\title{
2 Investigation of laminar burning velocities and 3 cellular instability for dimethyl carbonate at 4 elevated pressures
}

State Key Laboratory of Fire Science, University of Science and Technology of China, Hefei, Anhui, 230026, PR China

1 The authors contributed equally to this work.

13 *Corresponding Author:

14 Yong Jiang (Prof. \& Ph. D).

15 State Key Laboratory of Fire Science, University of Science and Technology of

16 China, Hefei, Anhui 230026, China

17 Tel.: +86-551-63607827;

18 Fax number: +86-551-63601669;

19 E-mail: yjiiang@ustc.edu.cn (Yong Jiang)

20 


\section{Supplementary figures}

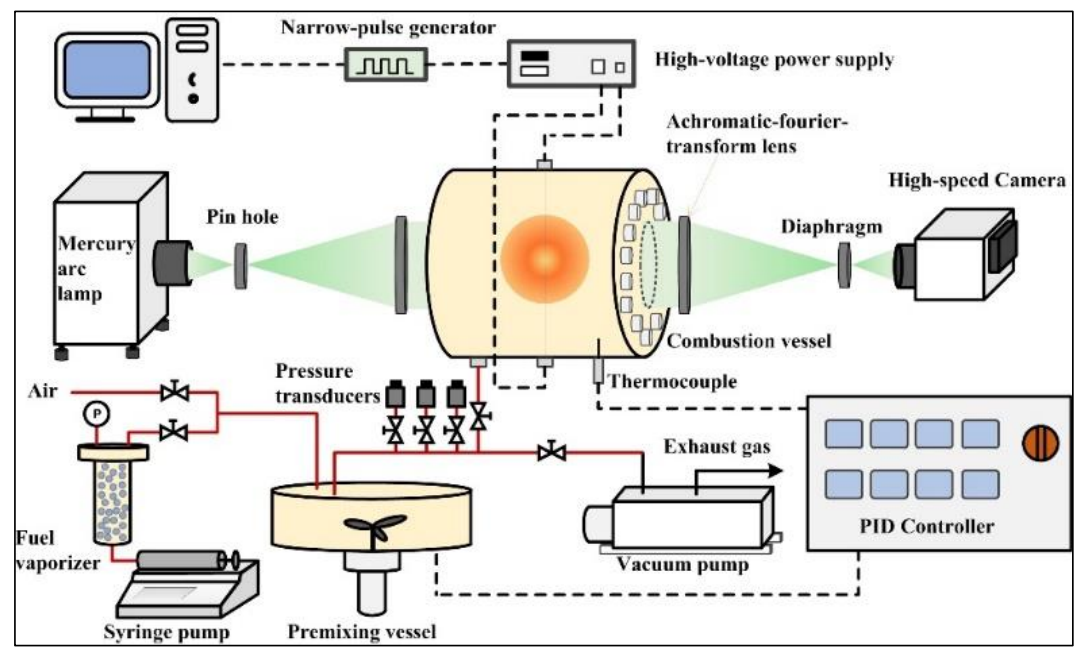

22 Fig. S1. Schematic diagram of the experimental apparatus, the heated pipelines and vessels are

23 marked in red and orange colors. 

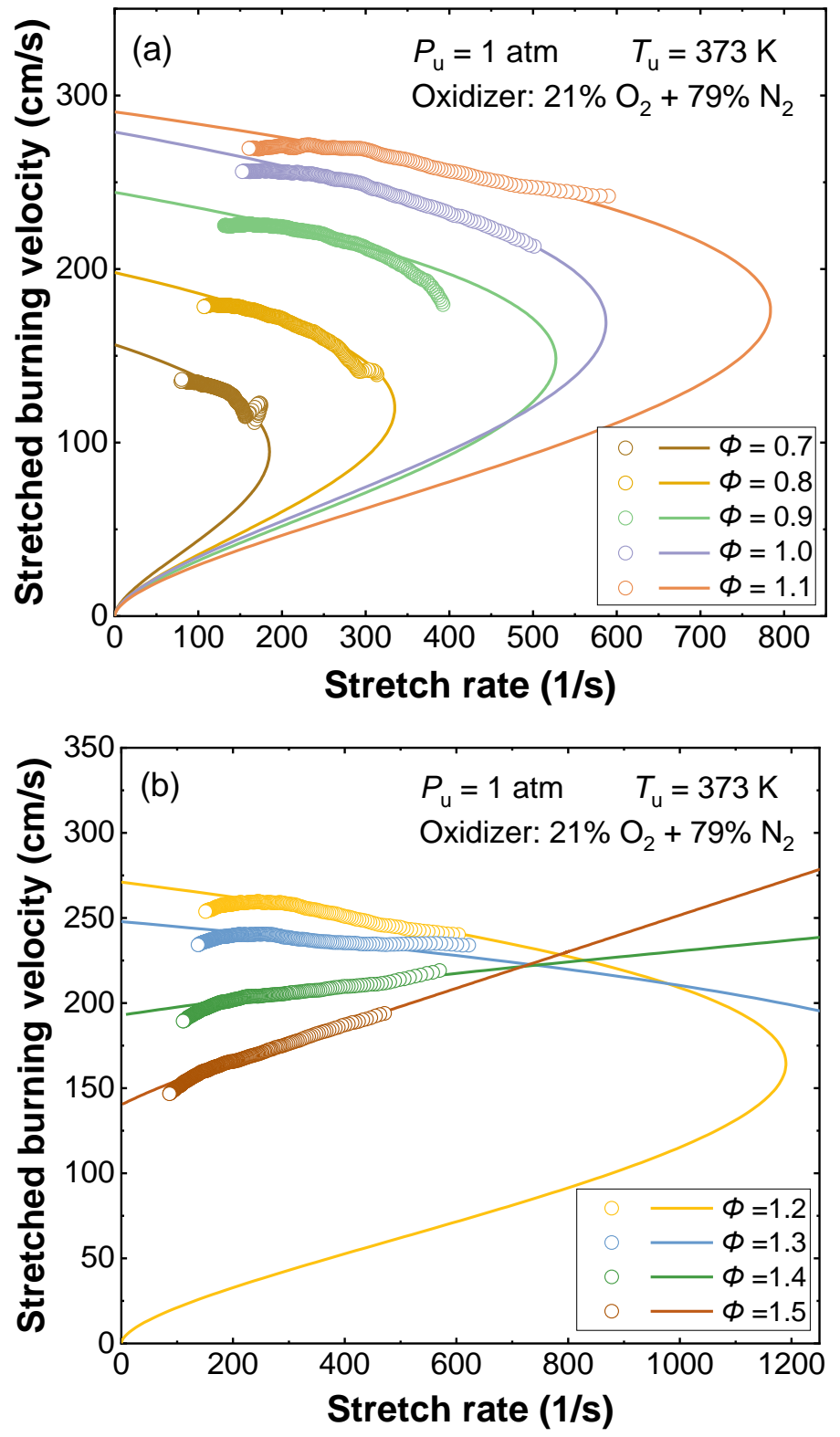

27 Fig.S2. Stretched burning velocities versus stretch rates at $373 \mathrm{~K}$ and $1 \mathrm{~atm}$ for $\mathrm{DMC} / \mathrm{O}_{2} / \mathrm{N}_{2}$

28 flames, over the equivalence ratio of 0.7-1.5. 

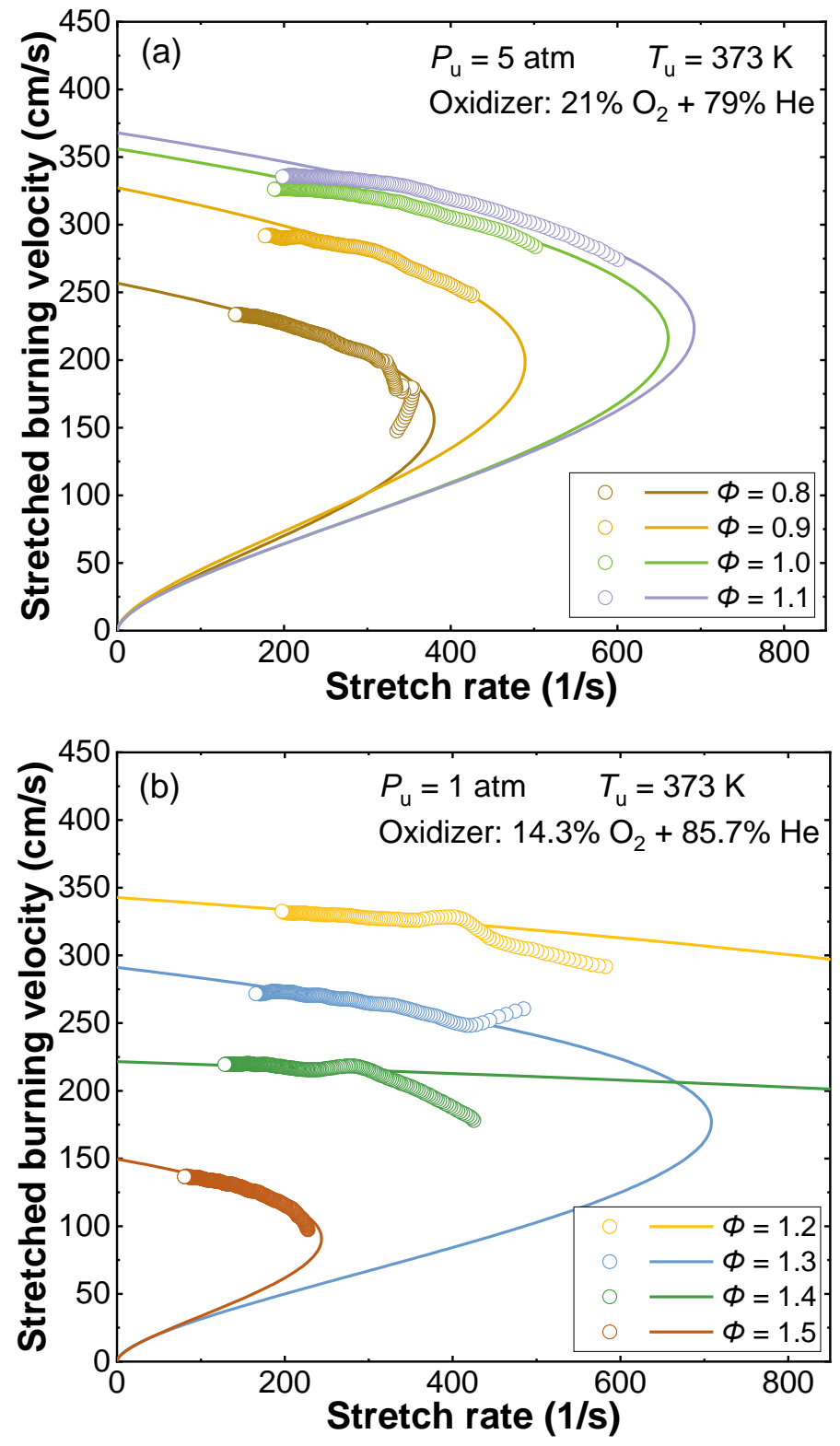

31 Fig.S3. Stretched burning velocities versus stretch rates at $373 \mathrm{~K}$ and 1 atm for $\mathrm{DMC} / \mathrm{O}_{2} / \mathrm{He}$

32 flames, over the equivalence ratio of $0.8-1.5$. 


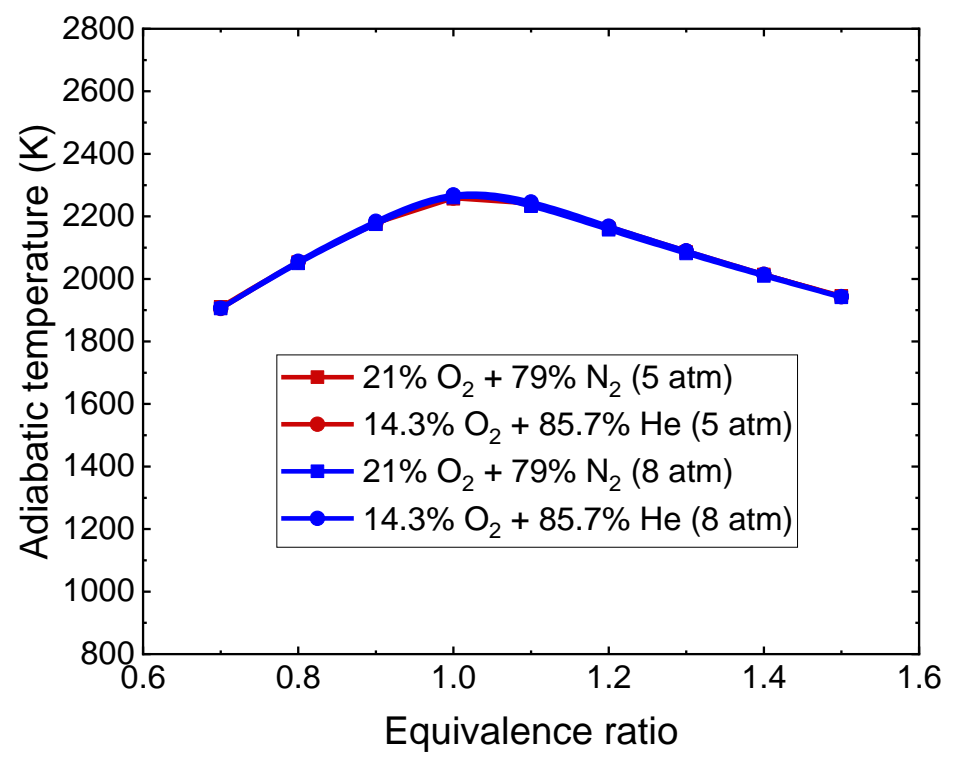

35 Fig. S4. The simulated adiabatic temperatures of DMC/21\% $\mathrm{O}_{2} / 79 \% \mathrm{~N}_{2}$ and $\mathrm{DMC} / 14.3 \% \mathrm{O}_{2} / 85.7 \% \mathrm{He}$ at $36 \quad 373 \mathrm{~K}$ and 5 and $8 \mathrm{~atm}$.

\section{The effective Lewis number}

The effective Lewis number $\left(L e_{e f f}\right)$, a weighted average of the Lewis numbers of the

39 "two" reactants (fuel blend/oxidizer), is evaluated as ${ }^{1-3}$ :

$$
L e_{e f f}= \begin{cases}\frac{L e_{o}+(1-\widehat{\Phi}) L e_{f}}{2-\widehat{\Phi}} & \Phi<1 \\ \frac{L e_{f}+(1+\widehat{\Phi}) L e_{o}}{2+\widehat{\Phi}} & \Phi>1\end{cases}
$$

$$
\widehat{\Phi}=Z e(\Phi-1)
$$

$$
L e_{i}=\frac{D_{T}}{D_{A B}}=\frac{\lambda}{\rho_{u} C_{p} D_{A B}}
$$

$$
Z e=E a\left(T_{a d}-T_{u}\right) /\left(R T_{a d}^{2}\right)
$$

$$
E a=-2 R\left[\partial \ln \left(\rho_{u} s_{u}^{0}\right)\right]
$$

Where the $L e_{e f f}$ is the effective Lewis number, $L e_{o}$ and $L e_{f}$ represent the Lewis

46 numbers of the oxidizer and fuel (calculated by equation (3)), respectively. Ze is

47 Zeldovich number, with $E a$, the overall active energy and $\mathrm{R}$, the gas constant. $T_{u}$

48 represents the initial temperature. The basic parameters, such as the density of the unburned 
49 mixture $\left(\rho_{u}\right)$, thermal conductivity of the mixture $(\lambda)$, specific heat capacity $\left(\mathrm{C}_{p}\right)$, 50 molecular diffusion coefficient $\left(D_{A B}\right)$, adiabatic flame temperature $\left(T_{a d}\right)$ and laminar

51 burning velocities $\left(s_{u}^{0}\right)$, are all calculated using the Chemkin-Pro software."

\section{Uncertainty analysis}

54 There have ever proposed the some mathematically methods for the estimation of

55 uncertainty for spherical flame expansion experiments ${ }^{4-7}$. However, among these methods,

56 the exact relationships between laminar burning velocities (LBVs) and independent

57 variables are needed for the calculation of the systematic uncertainties, which is hard to be

58 determined accurately and empirical parameters in the relationships are requisite.

59 The present work employed the method proposed by Moffat et al. ${ }^{8}$ and Wang et al. ${ }^{9}$

60 to determine the comprehensive uncertainties. Total uncertainties are consisted of

61 systematic uncertainties $\left(B_{S L}\right)$ and random or precision uncertainties $\left(P_{S L}\right)$, given by Eq. (6):

$$
U_{S L}={\sqrt{B_{S L}{ }^{2}+\left(\left(t S_{S L}\right) / \sqrt{M}\right)^{2}}}^{2}
$$

63 The random uncertainty is calculated from several repeats in a fixed condition, $\mathrm{M}$ is the

64 repeated number of experiments in each condition, $\mathrm{S}_{\mathrm{SL}}$ represents the sample standard

65 deviation, and $t$ is the Student's t-distribution for $95 \%$ confidence and M-1 degrees of

66 freedom.

67 The systematic uncertainties of pressure and temperature aroused from the deviation

68 for pressure gauge and thermocouple, respectively, which are related to manufacturer- 
69 specified precisions. The relative uncertainties of the repeated measurements were

70 analyzed via statistical analysis ${ }^{8}$. The measuring accuracy of the temperature controller is

710.00125 F.S. in this work, with resolution of $1^{\circ} \mathrm{C}$, the over the measuring range of $0-800^{\circ} \mathrm{C}$.

72 Results showed that the combined standard uncertainty of temperature is $0.16 \%$ in the

73 combustion vessel and $0.44 \%$ in the combustion vessel. The pressure range of this

74 experiment is $1-8 \mathrm{~atm}$, the accuracy of the used transducers is \pm 0.2 F.S., with smallest

75 division of $0.1 \mathrm{kPa}$. Thus the relative uncertainty caused by pressure is $0.11 \% \sim 0.41 \%$.

76 Owing to the mixtures were prepared by partial pressure methods, uncertainty in agent

77 volume fraction was controlled by pressure device. The uncertainty of equivalent ratio is

78 related to pressure, temperature, reactant purity and real gas effect, as shown in Eq. (7):

$79 \Phi=A \frac{\partial_{\text {fuel }} P_{\text {fuel }} Z_{\text {oxidizer }} T_{\text {oxidizer }}}{\partial_{\text {oxidizer }} P_{\text {oxidizer }} Z_{\text {fuel }} T_{\text {fuel }}}$

80 In which $A=\left(n_{\text {air }} / n_{\text {fuel }}\right)_{\text {sto }}$, the subscript sto refers to the stoichiometric

81 condition. $\partial, z, P, T$ represents the gas purity, compressibility factor, pressure and

82 temperature, respectively. The relative uncertainty of the density ratio in the present work

83 is estimated to be $1 \%-2 \%$.

84 As discussed by Wang et.al ${ }^{9}$, the flowchart in the measurement of LBV using

85 spherically expanding flame is shown in Fig. S4. The relative uncertainty of each procedure

86 can be calculated and then combined to calculate the comprehensive uncertainty. 


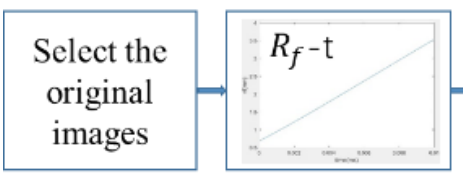

88

91 ignition and flame instability are negligible. The uncertainties of flame radiis and

92 propagation times are ignorable due to the high spatial resolution and recording speed.

93 Kelley et al. ${ }^{10}$ pointed out that the integration relationship of the nonlinear method can

94 eliminate the uncertainty of numerical differential calculation. The uncertainty of the

95 regression parameters over a wide range of equivalence has also been given in ${ }^{10}$, indicating

96 that the uncertainty near the stoichiometric flame in the $95 \%$ confidence interval is less

97 than $2 \%$.

98 The density ratio was calculated using chemical kinetic model by Chemkin-Pro

99 software. The hypothesis that replacing the burnt gas state with equilibrium state may cause

100 uncertainties, and the selection of different reaction mechanisms may also cause some

101 errors. Eq. (8) give the evaluation of the density ratio uncertainty which aroused from the

102 simplification and the kinetic model.

103

$$
\mathrm{u}\left(x_{i}\right)=\frac{R}{C \sqrt{n}}
$$




$$
\Delta\left(\delta_{i, \text { simplication }}\right)=\frac{\sigma_{S_{L}}-\sigma_{E_{q}}}{\sigma_{E_{q}}}
$$

105 In which $\mathrm{R}$ represents range of density ratio calculated by these mechanisms, $\mathrm{C}$ is the

106 coefficient of range, $\mathrm{n}$ represents the number of mechanisms (3 mechanisms were

107 discussed in this paper). The $\sigma_{E_{q}}$ is the density ratio calculated in equilibrium state, $\sigma_{S_{L}}$

108 is the density ratio calculated in premixed flame. Therefore, the relative uncertainty of the

109 density ratio in the present work is $1.2 \%-2 \%$.

110 The thermal radiation would directly reduce the flame temperature, and the cooling

111 effect would cause the inward flow in burned area. Both of above influences will reduce

112 the flame propagation velocity measured by schlieren method ${ }^{11,12}$. The uncertainty caused

113 by radiation effect were calculated by the method proposed by Yu et al. ${ }^{13}$, given in Eq. (10):

$$
B_{\text {rad,rel }}=\frac{\left(S_{u, R C F S}^{0}-S_{u, E x p}^{0}\right)}{S_{u, E x p}^{0}}=0.82\left(\frac{S_{u, E x p}^{0}}{S_{0}}\right)^{-1.44}\left(\frac{T_{u}}{T_{0}}\right)\left(\frac{P_{u}}{P_{0}}\right)^{-0.3}
$$

115 where $S_{u, R C F S}^{0}$ is the flame speed affected by radiation, $S_{u, E x p}^{0}$ represents the measured

116 burning velocity. Reference conditions are $S_{0}=1 \mathrm{~cm} / \mathrm{s}, T_{0}=298 \mathrm{~K}, P_{0}=1$ atm respectively.

117 The relative uncertainty of radiation effect is evaluated to be $1 \%-2.5 \%$ in this work.

118 Finally, the relative uncertainties of each component were combined and applied to

119 Eq. (1) for the calculation of the overall uncertainty. The total uncertainties of LBV in this

120 work is $3 \%-6 \%$. The uncertainties of equivalence ratio and laminar burning velocity are

121 finally presented in the figure as error bars. 


\section{Reference}

124 1. Matalon, M., Flame dynamics. Proceedings of the Combustion Institute 2009, 32, (1), $125 \quad 57-82$.

126 2. Wang, G.; Li, Y.; Li, L.; Qi, F., Experimental and theoretical investigation on cellular 127 instability of methanol/air flames. Fuel 2018, 225, 95-103.

128 3. Wang, X.; Fan, X.; Yang, K.; Wang, J.; Jiao, X.; Guo, Z., Laminar flame characteristics 129 and chemical kinetics of 2-methyltetrahydrofuran and the effect of blending with isooctane. 130 Combustion and Flame 2018, 191, 213-225.

131 4. Ravi, S.; Sikes, T. G.; Morones, A.; Keesee, C. L.; Petersen, E. L., Comparative study 132 on the laminar flame speed enhancement of methane with ethane and ethylene addition.

133 Proceedings of the Combustion Institute 2015, 35, (1), 679-686.

134 5. de Vries, J.; Lowry, W. B.; Serinyel, Z.; Curran, H. J.; Petersen, E. L., Laminar flame 135 speed measurements of dimethyl ether in air at pressures up to 10atm. Fuel 2011, 90, (1), 136 331-338.

137 6. W. Lowry, Z. S., G. Bourque, W. Metcalfe, J. de Vries, M. Krejci, H. Curran, E. 138 Petersen, Laminar flame speed measurements and modeling of pure alkanes and alkane 139 blends at elevated pressures. J. Eng. Gas Turb.Power 2011, 133, 091501.

140 7. Mei, B.; Zhang, X.; Ma, S.; Cui, M.; Guo, H.; Cao, Z.; Li, Y., Experimental and kinetic 141 modeling investigation on the laminar flame propagation of ammonia under oxygen 142 enrichment and elevated pressure conditions. Combustion and Flame 2019, 210, 236-246.

143 8. Moffat, R. J., Describing the uncertainties in experimental results. Exp. Therm Fluid 144 Sci 1988, 1, 3-17.

145 9. Wang, G.; Li, Y.; Yuan, W.; Zhou, Z.; Wang, Y.; Wang, Z., Investigation on laminar 146 burning velocities of benzene, toluene and ethylbenzene up to $20 \mathrm{~atm}$. Combustion and 147 Flame 2017, 184, 312-323.

148 10. Kelley, A. P.; Law, C. K., Nonlinear effects in the extraction of laminar flame speeds 149 from expanding spherical flames. Combustion and Flame 2009, 156, (9), 1844-1851.

150 11. Diévart, P.; Kim, H. H.; Won, S. H.; Ju, Y.; Dryer, F. L.; Dooley, S.; Wang, W.; 151 Oehlschlaeger, M. A., The combustion properties of 1,3,5-trimethylbenzene and a kinetic 152 model. Fuel 2013, 109, 125-136.

153 12. Hui, X.; Sung, C.-J., Laminar flame speeds of transportation-relevant hydrocarbons 154 and jet fuels at elevated temperatures and pressures. Fuel 2013, 109, 191-200.

155 13. Yu, H.; Han, W.; Santner, J.; Gou, X.; Sohn, C. H.; Ju, Y.; Chen, Z., Radiation-induced 156 uncertainty in laminar flame speed measured from propagating spherical flames. 157 Combustion and Flame 2014, 161, (11), 2815-2824. 